\title{
ANDRÉS BAUTiSTA-HERnÁEZ1: "REFLEXIONES EN TORNO A LA Regulación Jurídica de la Reducción del Riesgo de Desastre (Disaster Risk Reduction) y la Práctica Internacional al Respecto Seguida por la UNESCO".2
}

\section{"REMARKS ON LEGAL REGULATION OF DISASTER RISK REDUCTION. SPECIAL FOCUS ON THE UNESCO PRACTICE IN THIS FIELD".}

Resumen: La prevención constituye la etapa inicial del conjunto de fases de la gestión de catástrofes. A pesar de su importancia, la conceptualización de la prevención y dentro de ésta de la Reducción del Riesgo de Desastre, plantean numerosos interrogantes en nuestros días; del mismo modo, éstos se extienden a su regulación jurídica. Resulta también de interés la práctica internacional al respecto seguida por la UNESCO, organización por excelencia en materia de educación, ciencia y cultura, pero cuyo papel en el marco de la reducción del riesgo de desastres resulta poco conocido.

Abstract: Prevention represents the early steep of the phases in disaster management. Even though its importance, the idea of prevention, and within that, the Disaster Risk Reduction still raise various questions nowadays about its conceptualizations and its legal regulation. Also, may be of interest the international practice related to prevention within the UNESCO, the main international organization in education, science and culture, but its role related to Disaster Risk Reduction is rarely known.

Palabras clave: Catástrofe, Gestión de catástrofes, Práctica internacional, Prevención de catástrofes, Reducción del Riesgo de Desastre, RRD, UNESCO.

Keywords : Disaster, Disaster Management, Disaster Risk Reduction, DRR, Internacional Practice, Prevention of Disaster, UNESCO.

\footnotetext{
${ }^{1}$ Área de Derecho Internacional Público y Relaciones Internacionales de la Universidad de Málaga. Miembro del Centre de Droit International de Nanterre (París, Francia). abautista@uma.es

${ }^{2}$ Este trabajo se ha realizado en el marco del Interest Group on Biolaw, de la European Society of International Law-Societé Européenne de Droit International (ESIL-SEDI). El autor quiere hacer constar su agradecimiento a Alexandros Makarigakis, Especialista de programas de la UNESCO, por su apoyo en la búsqueda de documentación.
} 
ANDRÉS BAUTISTA-HERNÁEZ: "REFLEXIONES EN TORNO A LA REgUlACIÓN JURÍDICA DE LA REDUCCIÓN DEL RIESGO DE DESASTRE (DisASTER RISK REDUCTION) Y LA PRÁCTICA INTERNACIONAL AL RESPECTO SEGUIDA POR LA UNESCO”.

FECHA DE ENVÍO DE ORIGINAL: 18.02.2016 FECHA DE ACEPTACIÓN: 24.03.2016

DOI: http://dx.doi.org/10.12795/IETSCIENTIA.2016.i01.06

\section{INTRODUCCIÓN}

Hace poco más de un mes se aprobó el llamado «Acuerdo de París» ${ }^{3}$ en el marco del $21^{\text {er }}$ período de sesiones de la Conferencia de Estados Parte en la Convención Marco de las Naciones Unidas sobre el Cambio Climático (COP21) celebrada en París del 30 de noviembre al 11 de diciembre de 2015. Este Acuerdo se subsume en un texto que refleja tanto esperanzas de sus partidarios como decepciones de sus detractores ${ }^{4}$. Sin embargo, pese a este binomio de posiciones, un hecho objetivo late tras el mismo: la unanimidad de las Partes en determinar como problema común de la humanidad al cambio climático ${ }^{5}$.

En este contexto se hace aún más acuciante la necesidad de un análisis jurídico de la prevención de catástrofes, al igual que del conjunto de las que denominaremos "Fases de la Gestión de las Catástrofes" $"$. Todo ello a pesar de que la exigencia de avances en el desarrollo normativo e institucional en materia de Derecho Internacional de Catástrofes (DIC) ${ }^{7}$ se pusiera de manifiesto desde hace algún tiempo ${ }^{8}$.

\footnotetext{
${ }^{3}$ Hecho en París el 12 de diciembre de 2015, vid. NNUU, Aprobación del Acuerdo de París. Propuesta del Presidente. Proyecto de Decisión -/CP.21, Anexo, doc. NNUU FCCC/CP/2015/L.9/Rev.1, de 12.12.2015.

${ }^{4}$ Ya se observaban posicionamientos doctrinales escépticos antes de la propia celebración de la COP21, así vid.

v. gr. CAMPINS ERITJA, M., "De Kioto a París: ¿evolución o Involución de las Negociaciones Internacionales sobre el Cambio Climático?" en INSTITUTO ESPAÑOL DE ESTUDIOS ESTRATÉGICOS, Documento de Opinión 61/2015, 15.6.2015.

${ }^{5}$ Ibíd., pp. 1-2.

${ }^{6}$ Éstas hacen referencia al conjunto de actividades que pueden desarrollarse, en el marco del Derecho Internacional de Catástrofes para hacer frente a una catástrofe, y comprenderían la prevención, la preparación, la respuesta y la reconstrucción; vid. COPPOLA, D.-P., Introduction to International Disaster Management, Butterworth-Heinemann, 2006, p. 8; Propuesta de Decisión del Parlamento Europeo y del Consejo, relativa a un Mecanismo de Protección Civil de la Unión, doc. UE COM (2011) 934 final, pp. 2 y 5; BAUTISTAHERNÁEZ, A., «Recientes avances en la regulación de la Unión Europea en materia de catástrofes: el Mecanismo de Protección Civil de la Unión», en SÁNCHEZ PATRÓN, J.-M., TORRES CAZORLA, M.-I. y GARCÍA SAN JOSÉ, D.-I. (Coords.), Bioderecho, Seguridad y Medioambiente. Biolaw, Security and Environment, Tirant lo Blanch, Valencia, 2015, p. 184.

${ }^{7}$ Los límites de esta comunicación hacen imposible detenernos en la conceptualización del Derecho Internacional de catástrofes o de respuesta a las catástrofes, conocido en inglés, principalmente, como International Disaster Response Law (IDRL); sin embargo, sí puede señalarse que en la actualidad no puede hablarse de una unanimidad clara en la doctrina científica respecto de su determinación como rama de Derecho Internacional de nuevo cuño; señalamos a título de ejemplo y sin ánimo de exhaustividad algunas referencias que pueden ser de utilidad al lector sobre este extremo: MACALISTER-SMITH, P., «Disaster Relief: Reflections on the Role of International Law» en Heidelberg Journal of International Law (1985), p. 27; FISHER, D., Law and Legal Issues in International Disaster Response: A Desk Study, International Federation of Red Cross and Red Crescent Societies, 2007; HARPER, E. (Ed.), International Law and standards applicable
} 
ANDRÉS BAUTISTA-HERNÁEZ: "REFLEXIONES EN TORNO A LA REgUlACIÓN JURÍDICA DE LA REDUCCIÓN DEL RIESGO DE DESASTRE (DisASTER RISK REDUCTION) Y LA PRÁCTICA INTERNACIONAL AL RESPECTO SEGUIDA POR LA UNESCO”.

FECHA DE ENVÍO DE ORIGINAL: 18.02.2016 FECHA DE ACEPTACIÓN: 24.03.2016

DOI: http://dx.doi.org/10.12795/IETSCIENTIA.2016.i01.06

Igualmente, en este campo es fundamental establecer sinergias con otras áreas de conocimiento como son las ciencias naturales o exactas, o la física. Así, la teledetección satelital para los sistemas de alerta temprana (SAT), el desarrollo de modelos informáticos de inundaciones, el estudio geológico o hidrometeorológico, o la ingeniería civil son algunas disciplinas que adquieren un papel de primer nivel en materia de Reducción del Riesgo de Desastres (RRD), y con las que se trabaja en el seno de la UNESCO.

El siguiente trabajo trata de presentar al lector algunas notas respecto de la configuración jurídica de la prevención de catástrofes, en concreto de la RRD y las actuaciones sobre la materia seguidas por la UNESCO, como muestra de las sinergias existentes en materia de ciencia y derecho. Para ello, el mismo gira sobre dos ejes: en el primero (epígrafe 2) se exponen ideas respecto de la definición de prevención en materia de catástrofes y se estudia la Reducción del Riesgo de Desastre como elemento de ésta; en el segundo (epígrafe 3), se hace referencia a la práctica seguida en el ámbito internacional sobre

in natural disaster situations, IDLO, Roma, 2009, pp. 243-250; TORRES CAZORLA, M.-I., «La Comunicación de la Comisión Europea de 2010: Nuevas Medidas para Mejorar la Reacción de la UE en caso de Catástrofe», en PRIETO SANJUÁN, R.-A. y THOUVENIN, J.-M. (eds. académicos), Derecho Internacional y desastres: estudios sobre prevención y asistencia a las víctimas - Droit International et Catastrophes: études sur la prévention et l'assistance aux victimes - International Law and Disasters: Studies on Prevention and Assistance to Victims, Colección Estudios de Derecho Internacional, No ${ }^{\circ}$ 14, Pontificia Universidad Javeriana y Universidad París Ouest Nanterre-La Défense, Grupo Editorial Ibáñez, Bogotá, 2011; FISHER, D., «The Future of International Disaster Response Law», en German Yearbook of International Law, Vol. 55 (2012); DAVIES, S.E., «Natural Disasters and the Responsibility to Protect», en German Yearbook of International Law, Vol. 55 (2012); FERNÁNDEZ LIESA, CARLOS R. y OLIVA MARTÍNEZ, J.-D., El Derecho Internacional y la cooperación frente a los desastres en materia de Protección Civil, Dirección General de Protección Civil y Emergencias, Madrid, 2012; GUTTRY, A. de, GESTRI, M., VENTURINI, G. (Eds.), International Disaster Response Law, TMC Asser Press Springer, 2012, pp. 3-64; TORRES CAZORLA, M.-I., «Las catástrofes: una visión desde la regulación internacional y de la Unión Europea», en FORO PARA LA PAZ EN EL MEDITERRÁNEO, Actas de las VI Jornadas de Seguridad, Defensa y Cooperación. Estrategia Española de Seguridad y su Incidencia en el área mediterránea, Servicio de Publicaciones e Intercambio Científico de la Universidad de Málaga (SPICUM), Málaga, 2013; TORRES CAZORLA, M.-I., «Las Emergencias y Catástrofes como Riesgo para la Seguridad: una visión desde la perspectiva del Derecho Internacional Público a la luz de la Estrategia de Seguridad Nacional de Mayo de 2013» en ICADE, Revista Cuatrimestral de las Facultades de Derecho y Ciencias Económicas y Empresariales, No. 92 mayo-agosto (2014), pp. 77-106; CARON, D.-D, KELLY, M.-J., TELESETSKY, A. (Eds.), The International Law of Disaster Relief, Cambridge, 2014, en especial pp. 29 y ss.

${ }^{8}$ Ya en el marco de la Conferencia Mundial sobre la Reducción de los Desastres de Hyogo (2005) se pusieron de relieve las deficiencias institucionales, jurídicas y normativas, vid. Examen de la Estrategia y Plan de Acción de Yokohama para un mundo más seguro, doc. NNUU A/CONF.206/L.1, de 20.12.2004, p. 7 y ss.; COMISIÓN DE DERECHO INTERNACIONAL, Protección de las personas en caso de desastre, Memorando de la Secretaría, doc. NN.UU. A/CN.4/590, de 11.12.2007, p. 30. 
ANDRÉS BAUTISTA-HERNÁEZ: "REFLEXIONES EN TORNO A LA REgUlACIÓN JURÍDICA DE LA REDUCCIÓN DEL RIESGO DE DESASTRE (DisASTER RISK REDUCTION) Y LA PRÁCTICA INTERNACIONAL AL RESPECTO SEGUIDA POR LA UNESCO”.

FECHA DE ENVÍO DE ORIGINAL: 18.02.2016 FECHA DE ACEPTACIÓN: 24.03.2016

DOI: http://dx.doi.org/10.12795/IETSCIENTIA.2016.i01.06

RRD, centrándonos en el papel jugado por la UNESCO. Cierra el presente trabajo el epígrafe 4, dedicado a las principales conclusiones a las que se ha llegado tras su elaboración.

\section{APROXIMACIÓN AL CONCEPTO DE PREVENCIÓN Y SU REGULACIÓN JURÍDICA}

El desarrollo de la idea de prevención en materia de catástrofes tuvo sus inicios más destacados con la declaración del Decenio Internacional para la Reducción de los Desastres Naturales ${ }^{9}$, con la cual comenzaba un cambio en la tendencia general al sustituirse el interés en la situación post-catástrofe (respuesta) por la pre-catástrofe (prevención) ${ }^{10}$.

La idea de prevención ha estado ligada al desarrollo normativo del Derecho Internacional del Medio ambiente ${ }^{11}$. En este sentido se configuraba como un principio regulador de las catástrofes transfronterizas en materia de responsabilidad del Estado ${ }^{12}$. No obstante, esta obligación de prevención ha sido interpretada por parte de la doctrina como

\footnotetext{
9 A través de la Resolución AGNU 44/236, de 22 de diciembre de 1989, Decenio Internacional para la Reducción de los Desastres Naturales. En este sentido vid. NICOLETTI, B., "The Prevention of Natural and Man-Made Disasters: What Duties for States?”, en GUTTRY, A. de, GESTRI, M., VENTURINI, G. (Eds.), International Disaster Response Law, TMC Asser Press Springer, 2012, p. 179.

10 Cambio que se reflejaría en la Estrategia de Yokohama de 1994; COMISIÓN DE DERECHO INTERNACIONAL, Protección... op. cit., p. 28. En el ámbito de la UNESCO, puede verse UNESCO, Disaster Preparedness and Mitigation. UNESCO's Role, Paris, 2007, disponible en: http://unesdoc.unesco.org/images/0015/001504/150435e.pdf (consultado por última vez el 11.2.2016, al igual que el conjunto de citas a páginas web de esta contribución), p. 5.

${ }^{11}$ Sobre el principio de prevención en materia de Derecho Internacional del Medioambiente es inexcusable la cita de la opinión separada del juez CANÇADO TRINDADE en el asunto Plantas de celulosa en el Río Uruguay (Argentina c. Uruguay), vid. ICJ, Case Concerning Pulp Mills on the River Uruguay, Judgment, 20 April 2010, disponible en: http://www.icj-cij.org/docket/files/135/15885.pdf. Sobre esta rama del Derecho Internacional puede verse en general: RODRIGUEZ CARRION, A.-J., Lecciones de Derecho Internacional Público, Tecnos, 2007, pp.479-485; GONZALEZ CAMPOS, J.-D., SÁNCHEZ RODRÍGUEZ, L.-O., SÁENZ DE SANTA MARIA, P.-A., Curso de Derecho Internacional Público, 4a edición, Thomson Civitas, 2008, pp.

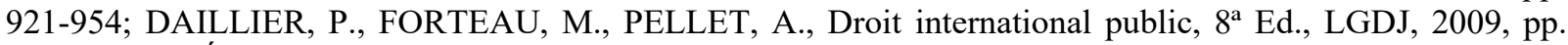
1417-1486; SÁENZ DE SANTA MARIA, P.-A., Sistema de Derecho Internacional Público, $2^{\mathrm{a}}$ Ed., Civitas, 2012, pp. 455-478; ABASS, A., International Law. Text, Cases, and Materials, Oxford University Press, 2012, pp. 609-646; CASADO RAIGÓN, R., Derecho Internacional, Tecnos, 2014, pp. 362-382. De manera específica, en nuestra doctrina vid. JUSTE RUIZ, J., La protección del medio ambiente en el ámbito internacional y en la Unión Europea, Valencia, Tirant lo Blanch, 2014, obra de referencia en la materia.

${ }^{12}$ Vid. NICOLETTI, B., «The Prevention...» op. cit., p. 179.
} 
ANDRÉS BAUTISTA-HERNÁEZ: "REFLEXIONES EN TORNO A LA REgUlACIÓN JURÍDICA DE LA REDUCCIÓN DEL RIESGO DE DESASTRE (DisASTER RISK REDUCTION) Y LA PRÁCTICA INTERNACIONAL AL RESPECTO SEGUIDA POR LA UNESCO”.

FECHA DE ENVÍO DE ORIGINAL: 18.02.2016 FECHA DE ACEPTACIÓN: 24.03.2016

DOI: http://dx.doi.org/10.12795/IETSCIENTIA.2016.i01.06

independiente y autónoma ${ }^{13}$; por tanto, sería aplicable al Derecho Internacional de Catástrofes ${ }^{14}$.

La prevención supone la primera de las "fases de gestión de catástrofes", en concreto la destinada al riesgo y la vulnerabilidad ${ }^{15}$, enmarcada dentro de lo que algunos han denominado las actividades de "gestión del riesgo de desastres"16. El "proceso de riesgo" juega un papel fundamental en la producción de una catástrofe, entendida como la concurrencia de: "the degree of exposure of people, infrastructure and economic activities to a physical event or hazard; and the vulnerability of those exposed to the hazard or shock" 17 . Se alinean por tanto la amenaza ${ }^{18}$ (un huracán o un terremoto) junto con la vulnerabilidad a ésta.

La prevención es un concepto poliédrico. En función de la faceta a la que nos aproximemos desvelará distintos significados y caracteres. De este modo, por un lado constituye una de las fases de la gestión en caso de catástrofe, por otro podría constituir un principio de carácter obligacional para los Estados. Así las cosas, son numerosas las definiciones ofrecidas de «prevención» hasta la fecha, y todas ellas giran en torno a la idea de

${ }^{13}$ Así, ROMANO, C.-P., «L'obligation de prévention des catastrophes industrielles et naturelles», en CARON, D.-D. y LEBEN, C. (Eds.), Les aspects internationaux des catastrophes naturelles et industrielles /The international aspects of natural and industrial catastrophes, The Hague Academy of International Law, Martinus Nijhoff, 2001, pp. 379-428.

${ }^{14}$ En este sentido NICOLETTI, B., «The Prevention...» op. cit., p. 179.

15 Entendida como las «Condiciones determinadas por factores o procesos físicos, sociales, económicos y ambientales que aumentan la susceptibilidad de una comunidad al impacto de amenazas», vid. ESTRATEGIA INTERNACIONAL PARA LA REDUCCIÓN DE DESASTRES/ NACIONES UNIDAS, Vivir con el riesgo: informe mundial sobre iniciativas para la reducción de los desastres, Naciones Unidas (ed.), Nueva York y Ginebra, 2004, p. 19.

16 Definida ésta como el "Conjunto de decisiones administrativas, de organización y conocimientos operacionales desarrollados por sociedades y comunidades para implementar políticas, estrategias y fortalecer sus capacidades a fin de reducir el impacto de amenazas naturales y de desastres ambientales y tecnológicos consecuentes", en ESTRATEGIA INTERNACIONAL PARA LA REDUCCIÓN DE DESASTRES/ NACIONES UNIDAS, Vivir... op. cit., p. 18.

${ }^{17}$ DEPARTAMENTO DE DESARROLLO INTERNACIONAL DE REINO UNIDO (DDIRU), Reducing the risk of disasters- helping to achieve sustainable poverty reduction in a vulnerable world: policy paper, Reino Unido, 2006, p. 5.

${ }^{18}$ Definida como «evento físico, fenómeno y/o actividad humana potencialmente perjudicial que puede causar la muerte o lesiones, daños materiales, interrupción de la actividad social y económica o degradación ambiental. Las amenazas incluyen condiciones latentes que pueden derivar en futuras amenazas/peligros, los cuales pueden tener diferentes orígenes: natural (geológico, hidrometeorológico y biológico) o antrópico (degradación ambiental y amenazas tecnológicas)» en ESTRATEGIA INTERNACIONAL PARA LA REDUCCIÓN DE DESASTRES/ NACIONES UNIDAS, Vivir... op. cit., p. 17. 
ANDRÉS BAUTISTA-HERNÁEZ: "REFLEXIONES EN TORNO A LA REgUlACIÓN JURÍDICA DE LA REDUCCIÓN DEL RIESGO DE DESASTRE (DisASTER RISK REDUCTION) Y LA PRÁCTICA INTERNACIONAL AL RESPECTO SEGUIDA POR LA UNESCO”.

FECHA DE ENVÍO DE ORIGINAL: 18.02.2016 FECHA DE ACEPTACIÓN: 24.03.2016

DOI: http://dx.doi.org/10.12795/IETSCIENTIA.2016.i01.06

actividades tendentes a evitar el riesgo de catástrofe ${ }^{19}$. De este modo y a efectos del presente trabajo, puede ofrecerse el siguiente concepto de prevención, indicando que será

«Aquella fase inicial del proceso de gestión de catástrofes en la que se llevan a cabo un conjunto de actuaciones encaminadas disminuir el riesgo asociado a una amenaza».

La prevención al igual que la preparación, tienen como objetivo reducir los efectos perjudiciales en caso de producirse el desastre. Así, por ejemplo, en el caso de una amenaza de origen natural (un desprendimiento de tierra), la prevención trabajará para reducir el riesgo de que el desprendimiento se produzca (con los consiguientes efectos dañinos para las personas, los bienes y el medioambiente), mediante técnicas de repoblación de árboles, o bien, estableciendo una zona de policía en la que no se podría construir.

Tomando como punto de partida la definición anterior, podemos incluir dentro de ésta la Reducción del Riesgo de Desastres ${ }^{20}$ (RRD), también conocida como Disaster Risk Reduction.

La RRD está integrada por una serie de elementos que pueden agruparse en dos grupos en función de que se trate de amenazas de tipo natural o de tipo antropogénico.

${ }^{19}$ ESTRATEGIA INTERNACIONAL PARA LA REDUCCIÓN DE DESASTRES/ NACIONES UNIDAS, Vivir... op. cit., p. 18. Inclusive, la viveza del asunto en materia conceptual se hace patente tras la creación del Grupo de trabajo intergubernamental de expertos de composición abierta sobre los indicadores y la terminología relacionados con la reducción del riesgo de desastres tras la recomendación realizada en la Tercera Conferencia Mundial sobre la Reducción del Riesgo de Desastres (párrafo 50 del Marco de Sendai para la Reducción del Riesgo de Desastres 2015-2030).

${ }^{20}$ Entendida como el «marco conceptual de elementos que tienen como función minimizar vulnerabilidades y riesgos en una sociedad, para evitar (prevención) o limitar (mitigación y preparación) el impacto adverso de amenazas[...]» (énfasis añadido); vid. ESTRATEGIA INTERNACIONAL PARA LA REDUCCIÓN DE DESASTRES/ NACIONES UNIDAS, Vivir... op. cit., p. 18. De igual modo es entendida como «Disaster risk reduction is the concept and practice of reducing disaster risks through systematic efforts to analyse and reduce the causal factors of disasters. Reducing exposure to hazards, lessening vulnerability of people and property, wise management of land and the environment, and improving preparedness and early warning for adverse events are all examples of disaster risk reduction», definición disponible en http://www.unisdr.org/who-weare/what-is-drr. 


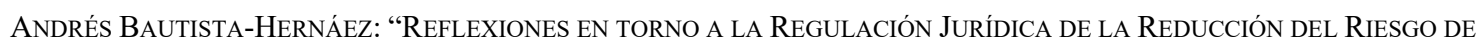
DESASTRE (DisASTER RISK REDUCTION) Y LA PRÁCTICA INTERNACIONAL AL RESPECTO SEGUIDA POR LA UNESCO”.

FECHA DE ENVÍO DE ORIGINAL: 18.02.2016 FECHA DE ACEPTACIÓN: 24.03.2016

DOI: http://dx.doi.org/10.12795/IETSCIENTIA.2016.i01.06

A) Respecto de las primeras, los distintos instrumentos tanto nacionales como internacionales sobre la materia recogen una serie de actividades o acciones a tomar que integrarían la fase de prevención, éstas serían las siguientes: la mejora de conocimientos sobre riesgos de catástrofes, la realización de actividades de enseñanza y sensibilización en prevención, la realización de evaluaciones de riesgos y vulnerabilidades, el establecimiento de sistemas de alerta temprana de catástrofes, el control de peligros que pudieran tener efectos transfronterizos, entre otras ${ }^{21}$. Igualmente, se han expresado como elementos de la prevención la cooperación internacional en materia de asistencia mutua, la investigación y el desarrollo, así como el intercambio de información o de tecnología.

B) En relación con las amenazas antropogénicas, debemos destacar la regulación contenida en el Convenio sobre los efectos transfronterizos de los accidentes industriales, hecho en Helsinki el 17 de marzo de $1992^{22}$, en el que se incluyen las siguientes medidas: «la fijación de objetivos en materia de seguridad, la adopción de disposiciones legales o de directrices sobre medidas y normas de seguridad, la identificación de actividades peligrosas específicas que exijan medidas preventivas especiales, la realización de evaluaciones de riesgos, la comunicación a las autoridades competentes de información pertinente, la aplicación de la tecnología más apropiada con el fin de prevenir esos accidentes, la prestación de educación y formación adecuadas, la exigencia de que se establezcan estructuras y prácticas eficaces de gestión, y la supervisión de las actividades peligrosas»» ${ }^{23}$.

Las normas jurídicas sobre RRD poseen un fuerte componente doméstico, es decir, es el derecho interno de los Estados el que regula de manera preeminente las cuestiones en esta materia. Ello es fruto de la importancia que se le ha venido dando al "fortalecimiento de

\footnotetext{
${ }^{21}$ Vid. ROMANO, C.-P., "L'obligation..." op. cit., p. 379; ESTRATEGIA INTERNACIONAL PARA LA REDUCCIÓN DE DESASTRES/ NACIONES UNIDAS, Vivir... op. cit., p. 18; COMISIÓN DE DERECHO INTERNACIONAL, Protección... op. cit., p. 33. Respecto de las actividades de la UE en el marco de la protección civil puede verse BAUTISTA-HERNÁEZ, A., "Recientes..." op. cit., pp. 185-186; y del mismo autor "La relación entre la Unión Europea y sus Estados miembros en la respuesta a las catástrofes: el papel de España en el mecanismo europeo de protección civil", comunicación a las XXVI Jornadas de la Asociación Española de Profesores de Derecho Internacional y Relaciones Internacionales "España y la Unión Europea en el orden internacional", 2015, en prensa.

${ }^{22}$ UNTS, Vol. 2105, Núm. 36605.

${ }^{23}$ Vid. Anexo IV del Convenio sobre los efectos transfronterizos de los accidentes industriales, hecho en Helsinki el 17 de marzo de 1992.
} 
ANDRÉS BAUTISTA-HERNÁEZ: "REFLEXIONES EN TORNO A LA REgUlACIÓN JURÍDICA DE LA REDUCCIÓN DEL RIESGO DE DESASTRE (DisASTER RISK REDUCTION) Y LA PRÁCTICA INTERNACIONAL AL RESPECTO SEGUIDA POR LA UNESCO”.

FECHA DE ENVÍO DE ORIGINAL: 18.02.2016 FECHA DE ACEPTACIÓN: 24.03.2016

DOI: http://dx.doi.org/10.12795/IETSCIENTIA.2016.i01.06

capacidades nacionales" sobre todo en países con menor desarrollo económico y/o con mayor tendencia a sufrir este tipo de fenómenos ${ }^{24}$. A pesar de la anterior afirmación, cada vez se está concediendo mayor importancia a la coordinación a nivel regional y global en materia de prevención de catástrofes, como puede verse en el nuevo Marco de Sendai que establece como una de sus siete metas mundiales

"Mejorar considerablemente la cooperación internacional para los países en desarrollo mediante un apoyo adecuado y sostenible que complemente las medidas adoptadas a nivel nacional para la aplicación del presente Marco para 2030”25.

A la hora de recoger por parte de los instrumentos internacionales las normas sobre prevención, pueden establecerse dos grupos: aquellos que regulan directamente la prevención $\mathrm{y}$ aquellos que lo hacen de forma indirecta o conexa ${ }^{26}$. Por un lado, nos encontramos con aquellos instrumentos que al regular aspectos de la gestión de catástrofes o de la respuesta, tienen disposiciones directamente relacionadas con la prevención o algunos de sus elementos; por otro, aquellos que regulando otras materias, indirectamente recogen ideas relacionadas con la prevención. Ejemplo de este último supuesto puede verse en la Convención sobre el derecho de los usos de los cursos de agua internacionales para fines distintos de la navegación de 21 de mayo de $1997^{27}$, en cuyo artículo 27 se demandaba a los Estados la toma de "medidas apropiadas para prevenir o mitigar las condiciones [...] que sean resultado de causas naturales o de un comportamiento humano [...] que puedan ser perjudiciales para otros Estados del curso de agua". Podemos encontrar ejemplos del primer grupo (es decir, relacionados directamente con la temática que nos ocupa) en el Convenio de Tampere de $1998^{28}$, el Convenio Marco de $2000^{29}$ o en la Decisión de la Unión Europea 1313/2013 ${ }^{30}$; en

\footnotetext{
${ }^{24}$ Como pudo verse en la Prioridad de Acción 1 del Marco de Acción de Hyogo, vid. Informe de la Conferencia Mundial sobre Reducción de los Desastres, Kobe, Hyogo (Japón), 18 al 22 de enero de 2005, doc. NNUU A/CONF.206/6, de 16.3.2005, p. 11.

${ }^{25}$ Vid. Resolución AGNU 69/283, de 3 de junio de 2015, Marco de Sendai para la Reducción del Riesgo de Desastres, doc. NNUU A/RES/69/283, de 23.6.2015, p. 7.

${ }^{26}$ COMISIÓN DE DERECHO INTERNACIONAL, Protección... op. cit., p. 31.

${ }^{27}$ Aprobada por la Resolución AGNU 51/229, doc. NNUU A/RES/51/229, de 8.7.1997, Anexo.

${ }^{28}$ Convenio de Tampere sobre el suministro de recursos de telecomunicaciones para la mitigación de catástrofes y las operaciones de socorro en caso de catástrofe, de 18 de junio de 1998, UNTS, vol. 2296, núm. 40906.

${ }^{29}$ Convenio Marco de asistencia en materia de protección civil, de 22 de mayo de 2000, UNTS., Vol. 2172, núm. 38131.
} 


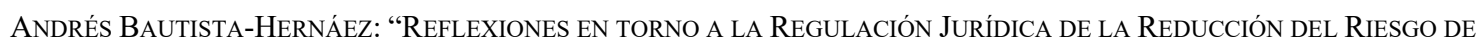
DESASTRE (DisASTER RISK REDUCTION) Y LA PRÁCTICA INTERNACIONAL AL RESPECTO SEGUIDA POR LA UNESCO”.

FECHA DE ENVÍO DE ORIGINAL: 18.02.2016 FECHA DE ACEPTACIÓN: 24.03.2016

DOI: http://dx.doi.org/10.12795/IETSCIENTIA.2016.i01.06

los supuestos anteriores se establecen, como elemento adicional, obligaciones de mejora de la coordinación y la cooperación en materia de prevención de catástrofes ${ }^{31}$.

\section{LA PRÁCTICA DEL SISTEMA DE NACIONES UNIDAS EN MATERIA DE REDUCCION DE RIESGO DE DESASTRES}

\subsection{LA LABOR DE NACIONES UNIDAS Y OTRAS ORGANIZACIONES}

Naciones Unidas tiene una posición de privilegio en la gestión de catástrofes a nivel universal ${ }^{32}$. Incluso podría considerarse que a esta función hace referencia el Artículo 1 de la Carta $^{33}$ al hablar de los propósitos de "mantener la paz y seguridad internacionales" o el de "realizar la cooperación internacional en la solución de problemas internacionales de carácter económico, social, cultural o humanitario, y en el desarrollo y estímulo del respeto a los derechos humanos y a las libertades fundamentales de todos”.

A la función de NNUU en materia de RRD hace referencia el Marco de Acción de Sendai que destaca, para apoyar la implementación del mismo, la necesidad de coordinación entre las Naciones Unidas y las organizaciones internacionales, así como entre las propias entidades, fondos, programas y agencias especializadas del sistema onusiano ${ }^{34}$.

La propia naturaleza de la Reducción del Riesgo de Desastre hace que se vean afectados numerosas áreas o disciplinas tales como el cambio climático, la salud, las

\footnotetext{
${ }^{30}$ Decisión 1313/2013/UE, del Parlamento Europeo y del Consejo, de 17 de diciembre de 2013, relativa a un Mecanismo de Protección Civil de la Unión (DOUE L 347, de 20.12.2013).

${ }^{31}$ Vid. arts. 3 1) y 1 7) del Convenio de Tampere; arts. 1 d) y 4 del Convenio Marco; y, art. 1.1 de la Decisión 1313/2013/UE.

${ }^{32}$ En este sentido HADDOW, G., BULLOCK, J., COPPOLA, D., Introduction to Emergency Management, $3^{\mathrm{a}}$ Ed., Elsevier, 2008, p. 255.

${ }^{33}$ Carta de las Naciones Unidas, firmada en San Francisco en 1945.

${ }^{34}$ Vid. pfos. 48 a), b) y c) del Marco de Sendai para la Reducción del Riesgo de Desastres 2015-2030, contenido en el Anexo I de la Resolución AGNU 69/283, de 3 de junio de 2015, doc. NNUU A/RES/69/283, pp. $24 \mathrm{y}$ 25. Un listado de los entes integrantes del sistema de NNUU puede encontrarse en http://www.unsceb.org/directory.
} 
ANDRÉS BAUTISTA-HERNÁEZ: "REFLEXIONES EN TORNO A LA REgUlACIÓN JURÍDICA DE LA REDUCCIÓN DEL RIESGO DE DESASTRE (DisASTER RISK REDUCTION) Y LA PRÁCTICA INTERNACIONAL AL RESPECTO SEGUIDA POR LA UNESCO”.

FECHA DE ENVÍO DE ORIGINAL: 18.02.2016 FECHA DE ACEPTACIÓN: 24.03.2016

DOI: http://dx.doi.org/10.12795/IETSCIENTIA.2016.i01.06

emergencias humanitarias, la ingeniería civil, la educación, el desarrollo sostenible, etcétera ${ }^{35}$.

El trabajo de NNUU en materia de Reducción del riesgo de desastres se centraliza principalmente a través de la Estrategia Internacional de Reducción de Desastres de Naciones Unidas (EIRD) ${ }^{36}$, junto con su Oficina para la Reducción del Riesgo de Desastre ${ }^{37}$ (por sus siglas en inglés UNISDR).

La lista de programas, agencias, fondos y otros entes del sistema de NNUU que trabajan directa o indirectamente en la EIRD, es abultada, abarcando materias tales como la economía, la cultura, la ciencia, la educación o la salud ${ }^{38}$. Ello es fiel reflejo de la preocupación de la Organización por la cuestión de la reducción de las catástrofes. Un estudio, siquiera menor, de todos ellos ocuparía seguro la extensión de este trabajo, por lo que nos limitaremos a enunciar los entes más relevantes en la materia ${ }^{39}$. De este modo, nos encontramos con la Asamblea General ${ }^{40}$, la Comisión de Derecho Internacional ${ }^{41}$, la Oficina

\footnotetext{
${ }^{35}$ Vid. LUIJE, T., DRR related terminology in other disciplines - example of UNECE work, presentación al UNISDR Technical Briefing on Terminology, 4 de septiembre de 2015, Ginebra, p. 2.

${ }^{36}$ Adoptada por la AGNU en 1999, la Estrategia Internacional forma parte de la Secretaría de NNUU bajo un Representante Especial del Secretario General para la Reducción del riesgo de desastre. Tiene encomendadas, entre otras, las funciones de servir de punto focal en el sistema de Naciones Unidas (según Resolución AGNU 56/195, de 21 de diciembre de 2001, Estrategia Internacional de Reducción de Desastres, doc. NNUU A/RES/56/195, de 21.1.2002) y la de apoyar la implantación del Marco de Sendai para la Reducción del Riesgo de Desastres (párrafo 48.c) del Marco, vid. en Anexo II a la Resolución AGNU 69/283, de 3 de junio de 2015, Marco de Sendai para la Reducción del Riesgo de Desastres, doc. NNUU A/RES/69/283, de 23.6.2015); puede encontrarse más información sobre la EIRD en su página web: http://www.unisdr.org/.

${ }^{37}$ Ésta última realiza las labores de secretaría.

${ }^{38}$ Vid. COPPOLA, D.-P., Introduction to International Disaster Management, Butterworth-Heinemann, 2006, p. 454.

${ }^{39}$ Puede encontrarse un listado de los órganos, entidades, fondos, programas y agencias especializadas de las Naciones Unidas que trabajan actualmente en materia de Reducción del Riesgo de Desastre en: http://www.unisdr.org/partners/united-nations.

${ }^{40}$ A través de la formulación de numerosísimas Resoluciones, entre muchas otras A/RES/70/282 de 4 de agosto de 2015, A/RES/69/284 de 25 de junio de 2015, A/RES/69/283 de 23 de junio de 2015, A/RES/68/211 de 29 de enero de 2014, A/RES/67/231 de 10 de abril de 2013 o la A/RES/67/209 de 12 de marzo de 2013; un listado mucho más amplio de Resoluciones sobre RRD puede encontrarse en http://www.preventionweb.net/english/professional/resolutions/index.php?o=res date\&o2=DESC\&ps $=50 \&$ date $=\&$ date $=0 \&$ tracks $=670 \& x=10 \& y=8$.

${ }^{41}$ Con el estudio del tema "La protección de las personas en caso de desastre" desde 2006, (vid. doc. NNUU A/61/10, pfo. 257).
} 
ANDRÉs BAUTISTA-HERNÁEZ: "REFLEXIONES EN TORNO A LA REgulaCiÓN JURÍDICA DE LA REDUCCIÓN DEL RIESGO DE DESASTRE (DisASTER RISK REDUCTION) Y LA PRÁCTICA INTERNACIONAL AL RESPECTO SEGUIDA POR LA UNESCO”.

FECHA DE ENVÍO DE ORIGINAL: 18.02.2016 FECHA DE ACEPTACIÓN: 24.03.2016

DOI: http://dx.doi.org/10.12795/IETSCIENTIA.2016.i01.06

de Naciones Unidas para la Coordinación de Asuntos Humanitarios $\quad(\mathrm{OCHA})^{42}$, la $\mathrm{UNESCO}^{43}$, la Agencia Internacional de la Energía Atómica (IAEA) ${ }^{44}$, o la Organización Internacional del Trabajo (OIT), entre otros muchos.

Como puede verse, el trabajo de la familia onusiana en la materia no hace más que aumentar ${ }^{45}$, dada la importancia que se le brinda a la prevención, lo que en cualquier caso supone un reto a la coordinación efectiva y eficiente de los escasos recursos con que cuenta ésta.

\subsection{EL PAPEL DE LA UNESCO EN LA RRD}

Tal vez resulte extraño asociar con la prevención en materia de catástrofes a una organización focalizada en la educación o la cultura, como es el caso de la UNESCO ${ }^{46}$; sin embargo esta aproximación es totalmente legítima y razonable, como se expondrá en las líneas que siguen.

\footnotetext{
${ }^{42}$ De gran relevancia en esta materia pues tiene encomendada la coordinación de las acciones humanitarias en colaboración con actores nacionales o internacionales. En materia de RRD, la OCHA trabaja en la incorporación de la perspectiva de reducción de catástrofes a los programas para una respuesta humanitaria efectiva.

${ }^{43}$ Un estudio con mayor detalle sobre ésta será ofrecido más adelante en este trabajo.

44 A través de la cooperación técnica con Estados; vid. https://www.iaea.org/technicalcooperation/Home/index.html.

${ }^{45}$ Favoreciéndose el desarrollo institucional para dar cobijo a tales tareas; vid. en Examen de la Estrategia y Plan de Acción de Yokohama para un mundo más seguro, doc. NNUU A/CONF.206/L.1, de 20.12.2004, p. 7.

${ }^{46}$ La Organización de Naciones Unidas para la Educación, la Ciencia y la Cultura fue creada en Londres en 1945 con base en la solidaridad y humanidad y con el objetivo de conseguir la paz entre los pueblos. Sobre esta organización especializada de Naciones Unidas puede verse, entre otros, ASCHER, Ch., «The Development of Unesco's Programme», en International Organization, Núm. 1, Vol. IV, Febrero (1950), pp. 12-26; LAVES, W., THOMSON, Ch., Unesco, Purpose, Progress, Prospects, Indiana University Press, 1957; THOMAS, J., U.N.E.S.C.O., Gallimard, París, 1962; MYLONAS, D., La Genèse de l'UNESCO: La Conférence des Ministres Alliés de l'Education (1942-1945), Bruylant, Bruselas, 1976; MATHIEU, J.-L., Les institutions spécialisées des Nations Unies, Masson, París, 1977, pp. 219-220; KASHLEV, Y., «UNESCO within the System of International Cooperation», en International Affairs, (1982), pp. 109 y ss.; PARTAN, D.G., «United Nations Educational, Scientific and Cultural Organization», en Encyclopedia of Public International Law, Vol. 5 (1983), pp. 314 y ss.; VALDERRAMA, F., Historia de la UNESCO, Unesco, París, 1991; MONCLÚS, A., SABAN, C. , «Análisis de la creación de la Unesco», en Revista Iberoamericana de Educación, Núm. 12 (1996), pp. 137-190.
} 
ANDRÉS BAUTISTA-HERNÁEZ: "REFLEXIONES EN TORNO A LA REgUlACIÓN JURÍDICA DE LA REDUCCIÓN DEL RIESGO DE DESASTRE (DisASTER RISK REDUCTION) Y LA PRÁCTICA INTERNACIONAL AL RESPECTO SEGUIDA POR LA UNESCO”.

FECHA DE ENVÍO DE ORIGINAL: 18.02.2016 FECHA DE ACEPTACIÓN: 24.03.2016

DOI: http://dx.doi.org/10.12795/IETSCIENTIA.2016.i01.06

En el marco de una preocupación creciente en materia de RRD, se ha focalizado como objeto de atención la denominada "cultura de prevención" 47 . De este modo, se manifiesta de vital importancia la educación como factor de formación para afrontar las catástrofes por parte de la ciudadanía. En este sentido, la "educación en reducción de catástrofes", la concienciación pública, en definitiva, el fortalecimiento de una cultura de seguridad relacionada con las catástrofes, constituyen pilares sobre los que asentar el cambio de comportamientos sociales que permitirán alcanzar los objetivos de reducción de riesgo o vulnerabilidad de las sociedades.

Dentro de esta cultura de prevención, es dónde la UNESCO desarrolla principalmente sus actividades sobre RRD, apoyando a sus Estados Miembros en la cartografía de amenazas naturales y vulnerabilidades, en la integración de la perspectiva de reducción de catástrofes en sus sistemas educativos y gestionando planes de RRD en los lugares que tienen la consideración de patrimonio mundial ${ }^{48}$, entre otros.

Si bien no se trata de un rol principal y diferenciado de la Organización, sus actuaciones en materia de catástrofe están íntimamente ligadas a sus esferas de actuación (educación, ciencia y cultura), constituyendo una preocupación transversal a todas ellas. A continuación se expondrán de manera resumida las principales actuaciones en materia de RRD en función de los sectores (siguiendo la nomenclatura de la UNESCO) en las que se desarrollen ${ }^{49}$.

A nivel general, debe señalarse que los textos fundamentales de la $\operatorname{UNESCO}^{50}$ no hacen ninguna referencia expresa a la Reducción del Riesgo de Desastre. Entre otras, la

\footnotetext{
${ }^{47}$ Vid. "Chapter 7: A culture of Prevention and Resilience?", en UNISDR, Making Development Sustainable: The Future of Disaster Risk Management. Global Assessment Report on Disaster Risk Reduction, Ginebra, 2015.

48 vid. UNITED NATIONS SYSTEM CHIEF EXECUTIVES BOARD FOR COORDINATION (CEB), United Nations Plan of Action on Disaster Risk Reduction for Resilience, 2013, disponible en http://www.preventionweb.net/files/33703_actionplanweb14.06cs1.pdf.

${ }^{49}$ El trabajo de la Organización se desarrolla y ejecuta a través de sus Sectores, Oficinas regionales, y centros de categoría I y II.

${ }^{50}$ Las principales normas que regulan la UNESCO son las siguientes: la Constitución de la Organización de las Naciones Unidas para la Educación, la Ciencia y la Cultura, aprobada en Londres el 16 de noviembre de 1945 (en adelante, Constitución de la UNESCO); el Reglamento de la Conferencia General, aprobado por la
} 
ANDRÉS BAUTISTA-HERNÁEZ: "REFLEXIONES EN TORNO A LA REgUlACIÓN JURÍDICA DE LA REDUCCIÓN DEL RIESGO DE DESASTRE (DisASTER RISK REDUCTION) Y LA PRÁCTICA INTERNACIONAL AL RESPECTO SEGUIDA POR LA UNESCO”.

FECHA DE ENVÍO DE ORIGINAL: 18.02.2016 FECHA DE ACEPTACIÓN: 24.03.2016

DOI: http://dx.doi.org/10.12795/IETSCIENTIA.2016.i01.06

principal razón obedece a la imposibilidad de detallar todas las actividades, presentes y futuras, de las que se hace cargo la Organización. No obstante, sí se contempló la posibilidad de que trabajase en el tema desde la perspectiva de las ciencias naturales (uno de los 5 Grandes Programas ${ }^{51}$ de la UNESCO) al igual que de modo transversal como respuesta a crisis y situaciones de transición ${ }^{52}$. Ello se realizó a través de sendas Resoluciones de la Conferencia General ${ }^{53}$ en las que se autorizaba a la Directora General a aplicar los distintos planes de acción ${ }^{54}$.

En el marco de la Estrategia a Plazo Medio de ocho años (2014-21 $)^{55}$ y de un Programa y Presupuesto cuatrienal (2014-17) ${ }^{56}$, la UNESCO trabaja sobre la Reducción del Riesgo de Desastre en distintos sectores y programas, aunque esta inclusión programática no supone una verdadera novedad en su seno ${ }^{57}$. De este modo, la Organización participa del cambio de posicionamiento, desde la óptica post-catástrofe a la pre-catástrofe, ayudando a construir una cultura global de comunidades resilientes -si se nos permite la expresión- a través de disposiciones transversales e interdisciplinares ${ }^{58}$.

Conferencia General en su $3^{\text {a }}$ reunión; y el Reglamento del Consejo Ejecutivo, aprobado por el Consejo Ejecutivo en su $29^{\mathrm{a}}$ reunión.

${ }^{51}$ Los denominados «Major Programmes» (MP), que corresponden con los sectores de la UNESCO son: I. Educación, II. Ciencias Exactas y Naturales, III. Ciencias Humanas y Sociales; IV. Cultura y V. Comunicación e Información.

${ }^{52} \mathrm{Vid}$. al respecto http://www.unesco.org/new/en/unesco/themes/pcpd/natural-disaster-risk-reduction/.

${ }^{53} \mathrm{La}$ Conferencia General es el órgano plenario que reúne a todos los Estados Miembros de la organización, constituye uno de los tres órganos principales de la UNESCO, y su principal misión consiste en la determinación de la «orientación y la línea de conducta general» de ésta; vid. Artículo IV de la Constitución de la UNESCO.

${ }^{54}$ Entre las recientes destacamos: las Resoluciones $37 \mathrm{C} / 3$, relativa al Gran Programa I (extendida al bienio 2016-17 por la 38 C/91, pfo. 19) y la 37 C/21, relativa al Gran Programa II (extendida al bienio 2016-17 por la $38 \mathrm{C} / 92$, pfo. 13).

${ }^{55}$ Vid. documento UNESCO 37 C/4: Estrategia a plazo medio, 2014-2021, Paris, 2014; dicha estrategia fue aprobada por la Conferencia General en su $37^{\mathrm{a}}$ reunión (resolución 37 C/1 de la Conferencia General) y validada por el Consejo Ejecutivo en su 194ª reunión (decisión 194 EX/18).

${ }^{56}$ Vid. documento UNESCO 37 C/5: Programa y Presupuesto aprobados, 2014-2017, París, 2014.

${ }^{57}$ De hecho, ya en 2007 la Conferencia General abordaba este asunto, si bien desde la perspectiva de la preparación y la mitigación (vid. Resolución 21 aprobada por la Conferencia General en su 34 a sesión), en su $35^{\mathrm{a}}$ sesión ya se hacía mención expresa a la prevención y la reducción de desastres naturales y humanos (vid. Resolución 19 aprobada por la Conferencia General en su 35 sesión), y será a partir de 2011 cuando ya se utilice el término Reducción del Riesgo de Desastre (vid. las Resoluciones 36 C/Res.17, 37 C/Res.21 y 38 C/Res.17).

${ }^{58} \mathrm{Vid}$. en http://www.unesco.org/new/en/natural-sciences/special-themes/disaster-risk-reduction/. 
ANDRÉS BAUTISTA-HERNÁEZ: "REFLEXIONES EN TORNO A LA REgUlACIÓN JURÍDICA DE LA REDUCCIÓN DEL RIESGO DE DESASTRE (DisASTER RISK REDUCTION) Y LA PRÁCTICA INTERNACIONAL AL RESPECTO SEGUIDA POR LA UNESCO”.

FECHA DE ENVÍO DE ORIGINAL: 18.02.2016 FECHA DE ACEPTACIÓN: 24.03.2016

DOI: http://dx.doi.org/10.12795/IETSCIENTIA.2016.i01.06

Por sectores, y respecto al de Educación, la UNESCO se preocupa por la educación y sensibilización. Por un lado la enseñanza para la gestión del riesgo ante desastres (principalmente en países con mayor número de catástrofes) ${ }^{59}$. En particular relacionado con el desarrollo sostenible, la Organización trabaja en el fortalecimiento de la educación para el desarrollo sostenible a través del asesoramiento sobre políticas y la asistencia técnica ${ }^{60}$.

El sector de Ciencias Naturales constituye el de mayor peso en la labor de RRD, principalmente debido al fuerte impulso como productor, transmisor y coordinador de conocimiento científico de la Organización. Es aquí donde la UNESCO se marca como objetivo estratégico el Promover la cooperación científica internacional en torno a los problemas críticos para el desarrollo sostenible ${ }^{61}$, que se concreta en «la promoción de la reducción del riesgo de desastres naturales, en particular fortaleciendo las capacidades en materia de sistemas de alerta temprana y evaluaciones de tsunamis, y otros peligros relacionados con los océanos, las inundaciones y los desprendimientos de tierras, para reducir los riesgos y mejorar la preparación y la capacidad de adaptación» (énfasis añadido ${ }^{62}$. Con ello se espera una mejora en la RRD a través del fortalecimiento de los Sistemas de Alerta Temprana (SAT) ${ }^{63}$.

La cultura supone también otro de los baluartes de la organización en el que se llevan a cabo actividades relacionadas con la RRD. Principalmente se centra en la protección del patrimonio mundial en caso de catástrofes ${ }^{64}$.

\footnotetext{
${ }^{59}$ Por ejemplo, desde la oficina regional para América Latina y Caribe en Santiago de Chile se llevan a cabo tareas de educación para el desarrollo sostenible y para la gestión del riesgo ante desastres, dadas las especiales condiciones de la región, con un alto número de amenazas naturales (terremonos, tsunamis, o erupciones volcánicas), junto con condiciones de vulnerabilidad (alta pobreza, deficiencias en los servicios públicos educativos); vid. http://www.unesco.org/new/es/santiago/education/disaster-risk-management-education/.

${ }^{60}$ Vid. Gran Programa 1, Eje de acción 2, Resultado Esperado 9, UNESCO 37 C/5: Programa y Presupuesto aprobados, 2014-2017, París, 2014, p. 42. Vid. sobre la inclusión de postulados de la RRD en los curriculum educativos a SELBY, D. y KAGAWA, F., Disaster Risk Reduction in School Curricula: Case Studies from Thirty Countries, UNESCO y UNICEF, España, 2012, p. 10, disponible en: http://unesdoc.unesco.org/images/0021/002170/217036e.pdf.

${ }^{61}$ Resolución $37 \mathrm{C} / 21$.

${ }^{62}$ UNESCO 37 C/5: Programa y Presupuesto aprobados, 2014-2017, París, 2014, p. 107.

${ }^{63}$ En este ámbito, como se expone, poseen gran importancia los sistemas de alerta temprana en los que participa la UNESCO. Ésta asiste a sus Estados Miembros a fortalecer sus capacidades tecnológicas y científicas a través de la identificación, seguimiento y afrontamiento de amenazas. Igualmente les ayuda en la preparación mediante los SAT; vid. en http://www.unesco.org/new/en/natural-sciences/special-themes/disaster-risk-reduction/.

${ }^{64}$ Vid. ICCROM, ICOMOS, UICN, CENTRO DEL PATRIMONIO MUNDIAL DE LA UNESCO, «Gestión del riesgo de desastres para el Patrimonio Mundial: una herramienta de identificación, evaluación y reducción de
} 
ANDRÉS BAUTISTA-HERNÁEZ: "REFLEXIONES EN TORNO A LA REgUlACIÓN JURÍDICA DE LA REDUCCIÓN DEL RIESGO DE DESASTRE (DisASTER RISK REDUCTION) Y LA PRÁCTICA INTERNACIONAL AL RESPECTO SEGUIDA POR LA UNESCO”.

FECHA DE ENVÍO DE ORIGINAL: 18.02.2016 FECHA DE ACEPTACIÓN: 24.03.2016

DOI: http://dx.doi.org/10.12795/IETSCIENTIA.2016.i01.06

Aunque en menor medida, en materia de comunicación e información también encontramos actividades de la UNESCO en materia de Reducción del Riesgo de Desastres. En este ámbito la Organización trabaja para favorecer el acceso universal a la información con el objetivo de aplicar «estrategias participativas de reducción del riesgo de desastres» ${ }^{65}$.

Junto con el trabajo propio de la organización, la UNESCO participa en distintos Programas, Redes e Iniciativas relacionados con la Reducción del Riesgo de Desastre ${ }^{66}$, bien desde una faceta de dirección o a través de una participación activa en los mismos pero sin adoptar tareas de gestión o administración.

\section{A MODO DE CONCLUSIÓN}

Como cierre del presente trabajo, exponemos a continuación algunas conclusiones a modo de recapitulación de las páginas precedentes.

riesgos para el patrimonio», en UNESCO, Revista del Patrimonio Mundial, Núm. 74 diciembre (2014), pp. 4749; o de la misma publicación BOCCARDI, G., «La $3^{\text {a }}$ Conferencia Mundial sobre la Reducción del Riesgo de Desastres», pp. 38-41; http://whc.unesco.org/en/disaster-risk-reduction/.

${ }^{65}$ Vid. documento UNESCO 37 C/5: Programa y Presupuesto aprobados, 2014-2017, París, 2014, p. 212.

${ }^{66}$ Alguno de los cuales son los siguientes: La Red Africana de Instituciones en Ciencia de la Tierra (ANESI), el Programa Future Earth, la Alianza Global para la Reducción del Riesgo de Desastres y la Resiliencia en el Sector Educativo (GADRRRES), el Sistema Mundial de Sistemas de Observación de la Tierra (GEOSS), el Modelo Global de Terremotos (GEM), la Red Mundial de Geoparques (GGN), el Sistema Mundial de Observación de los Océanos (GOOS), la Comisión Oceanográfica Intergubernamental de la UNESCO (COI), el Consorcio Internacional sobre Deslizamientos de Tierras (ICL), la Iniciativa Internacional sobre Sequías (IDI), la Iniciativa de la UNESCO sobre la ingeniería, la Iniciativa Internacional sobre Inundaciones (IFI), el Programa Internacional de Ciencias de la Tierra (PICG), el Programa Hidrológico Internacional (PHI), la Plataforma Intergubernamental Científico-Normativa sobre Diversidad Biológica y Servicios de los Ecosistemas (IPBES), la Plataforma Internacional para la Reducción de Desastres Sísmicos (IPRED), la Iniciativa Internacional sobre la Sedimentación (ISI), el Programa sobre el Hombre y la Biosfera (MAB), el Programa Gestión de las Transformaciones Sociales (MOST), la Alianza para el Ambiente y la Reducción del Riesgo de Desastres (PEDRR), la Reducción de las Pérdidas Causadas por Terremotos en la Región del Mediterráneo Oriental (RELEMR), la Reducción de las Pérdidas Causadas por Terremotos en la Región del Sur de Asia y Asia Central (RELSAR), la Reducción de las Pérdidas Causadas por Terremotos en la Región de Asia Central (RELCAR), la Iniciativa de la UNESCO para la Educación de Ciencias de la Tierra en África (ESEIA), el Centro del Patrimonio Mundial (WHC); vid. UNESCO, Reducción del Riesgo de Desastres (RRD): Contribución de la UNESCO frente al desafío global, 2015, p. 6 . 
ANDRÉS BAUTISTA-HERNÁEZ: "REFLEXIONES EN TORNO A LA REgUlACIÓN JURÍDICA DE LA REDUCCIÓN DEL RIESGO DE DESASTRE (DisASTER RISK REDUCTION) Y LA PRÁCTICA INTERNACIONAL AL RESPECTO SEGUIDA POR LA UNESCO”.

FECHA DE ENVÍO DE ORIGINAL: 18.02.2016 FECHA DE ACEPTACIÓN: 24.03.2016

DOI: http://dx.doi.org/10.12795/IETSCIENTIA.2016.i01.06

Primero. Las sinergias entre ciencia y la Reducción del Riesgo de Desastre son de vital importancia, como ya se pusiera de manifiesto en el Marco de Sendai, en el que al hablar de las funciones de los actores pertinentes se reclamaba que «El sector académico y las entidades y redes científicas y de investigación deben [...] apoyar la interacción entre las políticas y la ciencia para la toma de decisiones» ${ }^{67}$.

Segundo. Dentro de la prevención, pero extrapolable a todas las fases de la gestión de la catástrofe, es necesario que exista una coordinación y una armonización de las fuerzas en la misma dirección. Ya lo indicó la Asamblea General, cuando reiteraba «[...] encarecidamente que haya una coordinación y una coherencia efectivas, según proceda, entre los procesos intergubernamentales, como el Marco de Sendai para la Reducción del Riesgo de Desastres 2015-2030, la agenda para el desarrollo después de 2015 y otros procesos pertinentes a fin de crear sinergias, así como la necesidad de una coordinación y una coherencia efectivas al respecto ${ }^{68}$. Esta llamada a la coordinación se realiza como reflejo de una tendencia de interrelaciones entre sujetos de la que no es ajeno el Derecho Internacional ${ }^{69}$.

Tercero. En materia tan transcendente como la prevención de catástrofes debería tenderse, como señalara el profesor ALCAIDE FERNÁNDEZ, hacia un Derecho Internacional sustentado no por el consentimiento de cada Estado sino por los intereses comunes de la comunidad internacional ${ }^{70}$; el interés de protección y defensa de la persona, los bienes y el medioambiente debe predominar frente a reduccionismos nacionales, y es tarea de todos, y en el caso de los juristas iusinternacionalistas ofrecer interpretaciones de la norma, cuando no su creación, tendentes a este objetivo. En este sentido, la UNESCO realiza

\footnotetext{
${ }^{67}$ Énfasis añadido, vid. Apartado V., pfo. 36(b) del Marco de Sendai para la Reducción del Riesgo de Desastres 2015-2030.

${ }^{68}$ La cursiva es nuestra; Párrafo 8 de la Resolución AGNU 69/284, de 3 de junio de 2015.

${ }^{69}$ Como demuestra la humanización del mismo; vid. al respecto GARCÍA SAN JOSÉ, D., "La prevención de daños medioambientales transfronterizos resultantes de actividades peligrosas: recientes desarrollos en Derecho Internacional”, en RODRÍGUEZ CARRIÓN, A.-J. y PÉREZ VERA, E., Soberanía del Estado y Derecho Internacional. Homenaje al profesor Juan Antonio Carrillo Salcedo, Secretariado de Publicaciones de la Universidad de Sevilla, Servicio de Publicaciones de la Universidad de Córdoba, Servicio de Publicaciones de la Universidad de Málaga, Madrid, 2005, Tomo I, pp. 661-662.

${ }^{70}$ ALCAIDE FERNÁNDEZ, J., "Orden público y Derecho Internacional: desarrollo normativo y déficit institucional”, en RODRÍGUEZ CARRIÓN, A.-J. y PÉREZ VERA, E., Soberanía... op. cit., p. 115.
} 


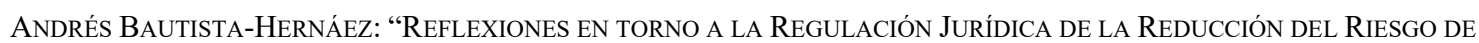
DESASTRE (DisASTER RISK REDUCTION) Y LA PRÁCTICA INTERNACIONAL AL RESPECTO SEGUIDA POR LA UNESCO”.

FECHA DE ENVÍO DE ORIGINAL: 18.02.2016 FECHA DE ACEPTACIÓN: 24.03.2016

DOI: http://dx.doi.org/10.12795/IETSCIENTIA.2016.i01.06

sus actividades desde esta óptica universalista basada en los postulados de humanidad, justicia, libertad y paz $^{71}$.

Cuarto. Las relaciones entre ciencia y derecho son evidentes en el trabajo que desarrolla la UNESCO en materia de RRD. La organización, avalada por su experiencia en el sector, promueve actuaciones encaminadas a luchar frente a las catástrofes especialmente en la fase de prevención. Sin embargo, se observa como en la configuración programática de la misma se confunden los términos y las políticas de cada fase de gestión de catástrofes. Si bien esta confusión difícilmente puede achacarse a una mala praxis cuando actualmente, en el seno de NNUU se sigue discutiendo respecto del alcance semántico de la terminología relacionada con catástrofes ${ }^{72}$.

En conclusión, el Derecho es una materia que lo abarca todo y a todos, y en concreto a la ciencia. Sin embargo, las respuestas de esta disciplina a las cuestiones relacionadas con las ciencias naturales o exactas, en muchos casos resultan «tardías e imperfectas» ${ }^{73}$. No obstante, esto no debe suponer sino un aliciente al académico para profundizar en los estudios y ofrecer mejores respuestas que las ya existentes a los viejos y nuevos problemas relacionados con el bioderecho.

\section{BIBLIOGRAFÍA}

ALCAIDE FERNÁNDEZ, J., “Orden público y Derecho Internacional: desarrollo normativo y déficit institucional”, en RODRÍGUEZ CARRIÓN, A.-J. y PÉREZ VERA, E., Soberanía del Estado y Derecho Internacional. Homenaje al profesor Juan Antonio Carrillo Salcedo, Secretariado de Publicaciones de la Universidad de Sevilla, Servicio de Publicaciones de la

\footnotetext{
${ }^{71}$ Como se recogen en el Preámbulo de su Constitución.

${ }^{72}$ Nos referimos al Grupo de trabajo intergubernamental de expertos de composición abierta sobre los indicadores y la terminología relacionados con la reducción del riesgo de desastres, creado en 2015.

${ }^{73}$ GARCÍA SAN JOSÉ, D., "Seguridad medioambiental y principio de necesidad en Bioderecho internacional", en SÁNCHEZ PATRÓN, J.-M., TORRES CAZORLA, M.-I. Y GARCÍA SAN JOSÉ, D.I. (Coords.), Bioderecho... op. cit., p. 132.
} 


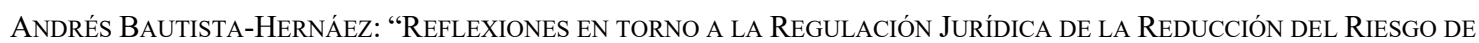
DESASTRE (DisASTER Risk REDUCTION) Y LA PRÁCTICA INTERNACIONAL AL RESPECTO SEGUIDA POR LA UNESCO”.

Universidad de Córdoba, Servicio de Publicaciones de la Universidad de Málaga, Madrid, 2005, Tomo I.

BAUTISTA-HERNÁEZ, A., «Recientes avances en la regulación de la Unión Europea en materia de catástrofes: el Mecanismo de Protección Civil de la Unión», en SÁNCHEZ PATRÓN, J.-M., TORRES CAZORLA, M.-I. y GARCÍA SAN JOSÉ, D.-I., (Coords.), Bioderecho, Seguridad y Medioambiente. Biolaw, Security and Environment, Tirant lo Blanch, Valencia, 2015.

CAMPINS ERITJA, M., “de Kioto a París: ¿Evolución o Involución de las Negociaciones Internacionales sobre el Cambio Climático?” en INSTITUTO ESPAÑOL DE ESTUDIOS ESTRATÉGICOS, Documento de Opinión 61/2015, 15.6.2015.

COPPOLA, D.-P., Introduction to International Disaster Management, ButterworthHeinemann, 2006.

DEPARTAMENTO DE DESARROLLO INTERNACIONAL DE REINO UNIDO (DDIRU), Reducing the risk of disasters- helping to achieve sustainable poverty reduction in a vulnerable world: policy paper, Reino Unido, 2006.

\section{ESTRATEGIA INTERNACIONAL PARA LA REDUCCIÓN DE DESASTRES/ NACIONES}

UNIDAS, Vivir con el riesgo: informe mundial sobre iniciativas para la reducción de los desastres, Edita Naciones Unidas, Nueva York y Ginebra, 2004.

GARCÍA SAN JOSÉ, D., “Seguridad medioambiental y principio de necesidad en Bioderecho internacional”, en SÁNCHEz PATRÓN, J.-M., TORREs CAZORLA, M.-I. y GARCÍA SAN JOSÉ, D.-I., (Coords.), Bioderecho, Seguridad y Medioambiente. Biolaw, Security and Environment, Tirant lo Blanch, Valencia, 2015.

GARCÍA SAN JOSÉ, D., "La prevención de daños medioambientales transfronterizos resultantes de actividades peligrosas: recientes desarrollos en Derecho Internacional", en 


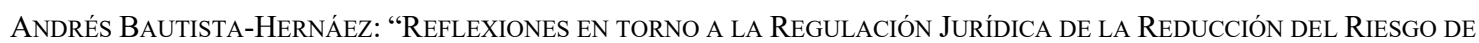
DESASTRE (DisASTER RISK REDUCTION) Y LA PRÁCTICA INTERNACIONAL AL RESPECTO SEGUIDA POR LA UNESCO”.

RODRÍGUEZ CARRIÓN, A.-J. y PÉREZ VERA, E., Soberanía del Estado y Derecho Internacional. Homenaje al profesor Juan Antonio Carrillo Salcedo, Secretariado de Publicaciones de la Universidad de Sevilla, Servicio de Publicaciones de la Universidad de Córdoba, Servicio de Publicaciones de la Universidad de Málaga, Madrid, 2005, Tomo I.

HADDOW, G., BULLOCK, J., COPPOLA, D., Introduction to emergency management, $3^{\mathrm{a}}$ Ed., Elsevier, 2008.

NICOLETTI, B., "The Prevention of Natural and Man-Made Disasters: What Duties for States?", en GUTTRY, A. de, GESTRI, M., VENTURINI, G. (Eds.), International Disaster Response Law, TMC Asser Press Springer, 2012.

ROMANO, C.-P., «L'obligation de prévention des catastrophes industrielles et naturelles», en CARON, D.-D. y LEBEN, C. (Eds.), Les aspects internationaux des catastrophes naturelles et industrielles /The international aspects of natural and industrial catastrophes, The Hague Academy of International Law, Martinus Nijhoff, 2001.

SELBY, D. y KAGAWA, F., Disaster Risk Reduction in School Curricula: Case Studies from Thirty Countries, UNESCO y UNICEF, España, 2012.

\section{UNITED NATIONS SYSTEM CHIEF EXECUTIVES BOARD FOR COORDINATION} (CEB), United Nations Plan of Action on Disaster Risk Reduction for Resilience, 2013, disponible en http://www.preventionweb.net/files/33703 actionplanweb14.06cs1.pdf.

"Chapter 7: A culture of Prevention and Resilience?", en UNISDR, Making Development Sustainable: The Future of Disaster Risk Management. Global Assessment Report on Disaster Risk Reduction, Ginebra, 2015. 